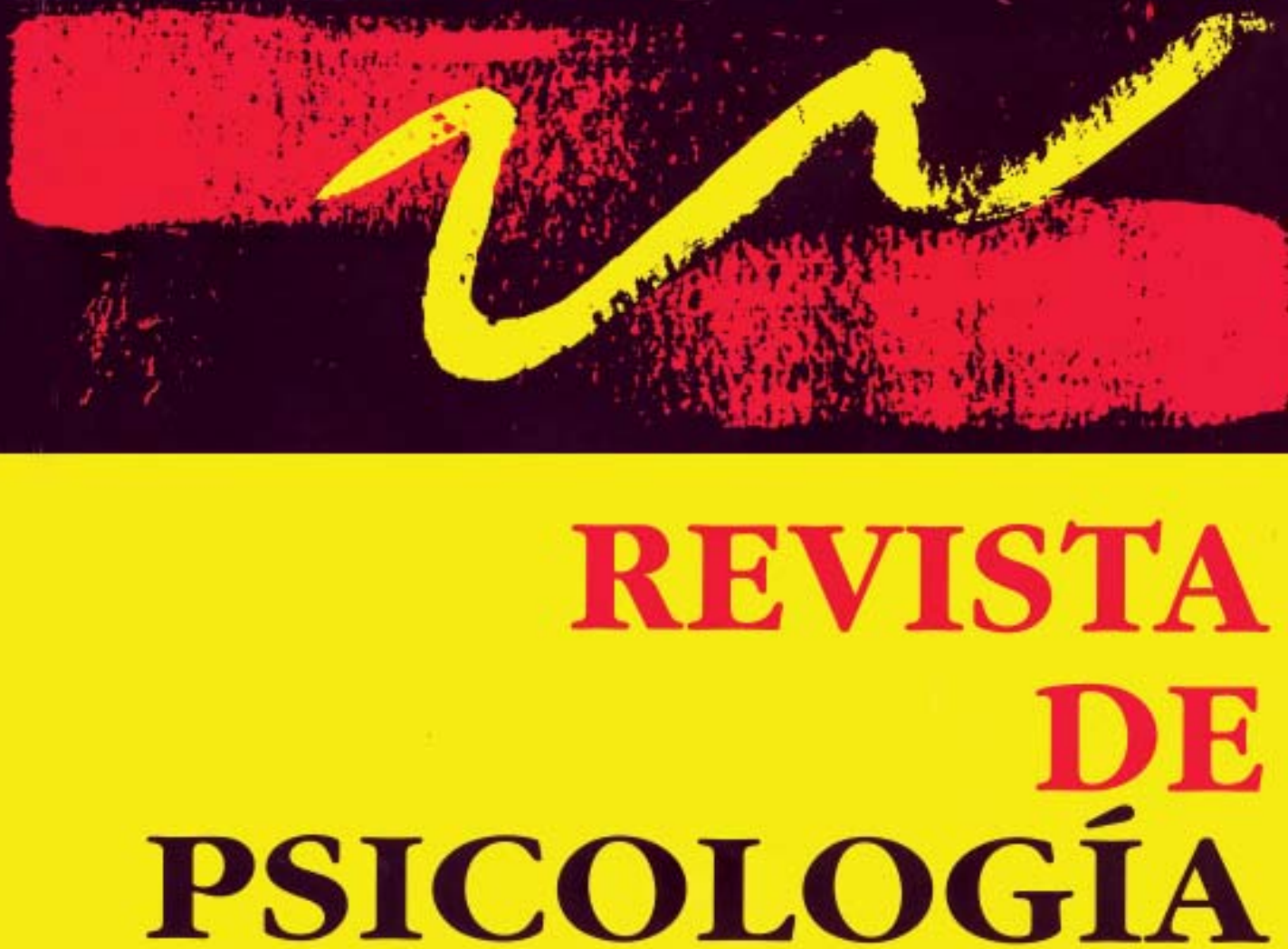

Silencio como secuela de la guerra interna en Perú

Comportamiento antisocial en menores escolares

e indigentes: influencia del vecindario y de los padres

La unidimensionalidad de un instrumento de medición: perspectiva factorial

Calidad de la amistad en niños de 8 a 10 años de una institución pública

Afrontamiento a la enfermedad crónica:

estudio en pacientes con insuficiencia renal crónica terminal
Javier Iguíñiz Echeverría

Ana Lilia Banda Castro y Martha Frías Armenta

Andrés Burga León

Doris Argumedo Bustinza y Carla Albornoz Álvarez

Mónica Cassaretto y Rosario Paredes

Reseña 


\section{REVISTA DE PSICOLOGÍA}

\section{Vol. XXIV. Primer semestre 2006 N $^{\circ} 1$ \\ CONTENIDO}

\section{ARTÍCULOS}

Javier Iguíniz Echeverría. Silencio como secuela de la guerra interna en Perú.

Ana Lilia Banda Castro y Martha Frías Armenta. Comportamiento antisocial en menores escolares e indigentes: influencia del vecindario y de los padres.

Andrés Burga León. La unidimensionalidad de un instrumento de medición: perspectiva factorial.

Doris Argumedo Bustinza y Carla Albornoz Álvarez. Calidad de la amistad en niños de 8 a 10 años de una institución pública.

Mónica Cassaretto y Rosario Paredes. Afrontamiento a la enfermedad crónica: estudio en pacientes con insuficiencia renal crónica terminal.

RESEÑA 


\title{
La unidimensionalidad de un instrumento de medición: perspectiva factorial
}

\author{
Andrés Burga León ${ }^{1}$ \\ Universidad Peruana Cayetano Heredia
}

\begin{abstract}
Este artículo explica qué es lo que implica la unidimensionalidad de un instrumento de medición. Para ello se presentan algunas definiciones y aportes teóricos sobre el tema. Luego, el análisis factorial es propuesto como uno de los métodos para evaluar la dimensionalidad de un instrumento de medición. Se señala como un problema importante el uso de las matrices de correlaciones de Pearson en los análisis factoriales a nivel de ítems. Estas correlaciones son problemáticas porque los ítems no cumplen con los supuestos necesarios para aplicar la correlación de Pearson: nivel de medición de intervalo y distribución normal de la variable. Como alternativa se postula y ejemplifica el uso de las correlaciones tetracóricas y policóricas.

Palabras clave: unidimensionalidad, Teoría Clásica de los Tests, análisis factorial, correlaciones tetracóricas, correlaciones policóricas.
\end{abstract}

\section{The unidimensionality of a measurement instrument: A factorial perspective}

This article explains what we mean by the unidimensionality of a measurement instrument, therefore we present some definitions and theoretical contributions about this subject. Factor analysis is proposed as one of the many methods for assessing the unidimensionality of a measurement instrument. The use of Pearson correlations matrices on item-level factor analysis is identified as an important problem. Those correlations are problematic because items didn't carry out the necessary assumptions in order to apply the Pearson correlation: interval-level measurement and normal distribution of the variable. As an alternative we propose and exemplify the use of tetrachoric and polychoric correlations.

Keywords: Unidimensionality, Classical Test Theory, factor analysis, tetrachoric correlations, polychoric correlations.

Docente de la UPCH. Licenciado en Psicología por la Universidad de Lima. Magíster en Psicología Educativa por la Universidad Peruana Cayetano Heredia. Consultor del LLECE (Laboratorio Latinoamericano de Evaluación de la Calidad Educativa). Coordinador del Equipo de Medición e Informática de la Unidad de Medición de la Calidad Educativa (UMC) del Ministerio de Educación. Áreas de investigación: psicometría, evaluación educativa, Psicología de la Personalidad. Correo electrónico: aburgal@minedu.gob.pe 

La unidimensionalidad implica que un sólo rasgo latente o constructo se encuentra en la base de un conjunto de ítems (Hattie, 1985). En otras palabras, un instrumento será unidimensional si las respuestas dadas a él son producidas basándose en un único atributo. Wright y Linacre (1989) señalan que, en la práctica, ningún instrumento puede ser perfectamente unidimensional. Lo que se busca es tener instrumentos que, en esencia, muestren unimensionalidad. Por ejemplo, muchos factores como la motivación, la ansiedad y la velocidad de respuesta tienen un impacto sobre el desempeño de una persona en un conjunto de ítems (Hambleton, Swaminathan \& Rogers, 1991). Lo importante es que un instrumento de medida represente con sus puntuaciones un solo factor dominante. Con esto lo que se quiere lograr es que la mayor cantidad de la varianza observada en las respuestas a los ítems sea explicada por un solo atributo latente (Embretson \& Reise, 2000).

Ésta no es la única manera que existe de entender la unidimensionalidad. Por ejemplo, Bejar (1983) sostiene que la unidimensionalidad no implica necesariamente que las respuestas a un conjunto de ítems se deban a un único proceso psicológico. Considera que puede haber un amplio conjunto de procesos psicológicos implicados en dichas respuestas, pero en la medida que los mismos procesos afecten de la misma forma las respuestas a un conjunto de ítems, se podrá sostener que existe una unidimensionalidad esencial en dicho instrumento de medida.

Es muy importante tener un instrumento unidimensional, ya que éste será para muchos un requisito indispensable para generar buenas medidas (Wright \& Masters, 1982; Wright \& Stone, 1998). Las puntuaciones obtenidas de la aplicación de un instrumento 
psicométrico, dentro de la Teoría Clásica de los Tests siguen un modelo monotónico lineal, es decir, se asume que existe una relación lineal entre el puntaje directo obtenido y el nivel del rasgo o tributo que se está midiendo. A más puntaje directo, más de ese rasgo o atributo posee la persona evaluada. ¿De dónde proviene ese puntaje directo o puntaje global? De la suma de los puntajes obtenidos en cada uno de los ítems. Como señala Cuesta (1996), el obtener los puntajes globales sumando las calificaciones de cada ítem supone que se está midiendo con ellos un solo constructo; de lo contrario, no habría ningún fundamento que soporte las operaciones aritméticas realizadas con los ítems. De la misma manera, si se pretende medir un nivel en una variable, ésta no debe estar «contaminada» por los niveles que posee la persona evaluada en otras variables (Stout, 1987 como se cita en Cuesta, 1996). Por lo tanto, evaluar la unidimensionalidad es un requerimiento muy importante en el desarrollo de instrumentos de medición.

Si bien no existe una única línea metodológica para evaluar la unidimensionalidad (Anderson, Gerbin \& Hunter, 1987; Linacre, 1994; Wright, 1994), presentaremos brevemente los aportes del análisis factorial como herramienta para evaluarla. Esto se debe a que éste sigue siendo una herramienta muy utilizada al momento de estudiar la dimensionalidad de un conjunto de ítems (Muñiz, 1997).

Un problema importante al que nos enfrentamos es que, dentro de la perspectiva factorialista, no hay un criterio unánime que nos sirva para afirmar que un instrumento de medición es básicamente unidimensional. Sin embargo, diversos autores (Carmines \& Zeller, 1979; Reckase, 1979; Zwick, 1985) proponen un conjunto de guías que pueden servirnos para determinar la unidimensionalidad de un test. Como veremos a continuación, la mayoría de ellos propone criterios que toman en cuenta la varianza explica- 
da por el primer factor extraído. Así, un conjunto de ítems será unidimensional si el primer factor explica por lo menos:

- $\quad$ el $40 \%$ de la varianza (Carmines \& Zeller, 1979),

- $\quad$ el $20 \%$ de la varianza (Reckase, 1979),

- $\quad$ entre el 17 y $40 \%$ de la varianza, usando matrices de correlaciones phi (Zwick, 1985),

- $\quad$ entre el $30 \%$ a $40 \%$ de la varianza, usando matrices de correlaciones tetracóricas (Zwick, 1985).

La cantidad de varianza explicada por el primer factor no es el único criterio que puede tomarse en cuenta para evaluar la unidimensionalidad de un instrumento. Hattie (1985) propone que un instrumento de medida será unidimensional si el cociente entre la diferencia del primer y segundo autovalor, y la del segundo y tercer autovalor, es mayor a 3. Por otro lado, Cuesta (1996) propone factorizar las puntuaciones obtenidas por cada persona en cada uno de los factores (denominados de primer orden), y si se obtiene un único factor de segundo orden, se podrá afirmar que nos encontramos básicamente frente a una medida unidimensional.

¿Cuál de todos estos criterios utilizar? En nuestra opinión, no es suficiente fijarnos sólo en uno de ellos. Especialmente creemos que el criterio de Reckase (1979), referido a la cantidad de varianza explicada por el primer factor extraído, resulta demasiado laxo. Asimismo, el uso de correlaciones phi tampoco es un buen criterio pues, como explicaremos más adelante, el utilizar este tipo de correlaciones en el análisis factorial tiene algunos problemas asociados a la formación de factores espurios. Además, no debemos dejar de lado otros aportes para evaluar la unidimensionalidad, especialmente aquellos provenientes de los modelos de análisis Rasch (Linacre, 1994; Wright, 1994) y de la Teoría de Respuesta al Ítem en general, como el análisis factorial 
de información total (Embretson \& Reise, 2000; Waller, 2001).

Al utilizar el análisis factorial como instrumento para evaluar la unidimensionalidad, no sólo nos encontramos frente al problema de la falta de acuerdo en los criterios para establecer si una medida es unidimensional. Otra dificultad muy importante lo encontramos en los supuestos mismos del análisis factorial. Tal y como se encuentra disponible en la mayoría de programas estadísticos, se factorizan matrices de correlaciones calculadas mediante el método de Pearson (Kubinger, 2003; O'Connor, s. f.). Este tipo de correlación asume que las variables están medidas a nivel de intervalo y tienen una distribución normal (Guilford \& Fruchter, 1984).

Un problema importante lo encontramos en la factorización de ítems binarios. Waller (2001) nos dice que el análisis factorial asume un modelo lineal en la regresión de la respuesta a un ítem sobre el puntaje factorial subyacente. La dificultad está en que, al momento de aplicar el modelo lineal con datos binarios, las personas con puntajes factoriales muy altos o muy bajos pueden tener probabilidades superiores a 1.00 o inferiores a 0.00 de «acertar» o «estar de acuerdo» con el ítem. Por otro lado, Cuesta (1996) nos dice que en el caso de que la variable sea dicotómica al momento de realizar un análisis factorial sobre una matriz de correlaciones, se aplica una modificación del coeficiente de Pearson, conocida como coeficiente $p h i$. En estos casos generalmente se forman factores espurios. Nunnally y Bernstein (1995) señalan que los ítems fáciles se juntarán con otros fáciles y los difíciles con otros difíciles; además, los de alto endosamiento formarán factores separados de los de bajo endosamiento, a pesar que los ítems en el fondo sean unidimensionales. Por este motivo, se les suele llamar factores de dificultad (Cuesta, 1996; Kubinger, 2003). Nosotros creemos que esta terminología es sólo aplicable a las pruebas de ejecución máxima. En el caso de las de ejecución típica, proponemos utilizar el tér- 
mino «factores de endosamiento».

Kubinger (2003) discute el problema de las correlaciones phi. Nos indica que los ítems dicotómicos constituyen escalas ordinales. Por ejemplo, acertar a un ítem implica más habilidad que fallarlo; estar de acuerdo con una afirmación como «me gusta asistir a fiestas» implica más extroversión que estar en desacuerdo con ella. Al utilizar el coeficiente phi, su valor máximo depende de los marginales (dificultad de los ítems). Se propone como solución el utilizar una matriz de correlaciones corregidas, en la que cada correlación phi se divide entre el valor máximo que puede asumir una correlación phi dada la dificultad del par de ítems que se están correlacionando (Cuesta, 1996). El problema para Kubinger (2003) es que no se conoce la distribución que este cociente presenta, por lo cual una mejor alternativa es utilizar correlaciones tetracóricas, las que asumen que, tras esas dicotomías, se encuentran dos variables distribuidas normalmente (Guilford \& Fruchter, 1984). Lo que ocurre es que un rasgo latente con distribución normal se ve dicotomizado en el momento de producirse la respuesta a un ítem. Es decir, las personas que tengan un nivel de rasgo latente por debajo del punto de corte del ítem (dificultad o endosamiento) lo fallarán o se mostrarán en desacuerdo con el mismo. En resumen, cuando uno emplea la matriz de correlaciones tetracóricas, como insumo para realizar un análisis factorial, se está haciendo una estimación mucho más precisa de la dimensionalidad de un conjunto de ítems.

En el caso de las escalas politómicas, nos encontramos realmente frente a medidas de tipo ordinal. Con las escalas politómicas, una estrategia que se emplea con frecuencia es calcular los índices de asimetría y curtosis, eliminando aquellos ítems que muestren una marcada distancia con respecto de los parámetros de la curva normal (Jiménez, Artiles \& Yáñez, 1997). Sin embargo, esta estrategia se basa en el cálculo de índices de asimetría y curtosis, que suponen el nivel de medición de intervalo de las variables. Como señalamos con anterioridad, este supuesto no se cumple a nivel de ítems. 
Lo más adecuado en estas situaciones es factorizar matrices de correlaciones policóricas que han sido desarrolladas especialmente para correlacionar dos variables ordinales, suponiendo que, en el fondo, se trata de variables de intervalo que presentan una distribución normal (O'Connor, s. f.; Waller, 2001). Se puede utilizar el programa informático LISREL para calcular las matrices de correlaciones y luego exportarlas a otros programas para realizar los análisis factoriales. Una ventaja de utilizar el LISREL es que provee, además, un conjunto de índices (Chi-cuadrado, RMSEA) que permite contrastar la hipótesis de una distribución normal bivariada para cada par de ítems correlacionados (Jöreskog, 2002).

En conclusión, si queremos factorizar ítems a fin de investigar la dimensionalidad de los mismos, debemos utilizar las herramientas estadísticas adecuadas: matrices de correlaciones tetracóricas, si tenemos ítems dicotómicos, y matrices de correlaciones policóricas, si tenemos ítems politómicos.

\section{Ejemplo para ítems dicotómicos}

A fin de ilustrar nuestro argumento a favor del uso de las correlaciones tetracóricas, presentaremos los análisis factoriales realizados a una de las pruebas aplicadas en el área de matemáticas en el EN2004. Dicha prueba está conformada por 21 ítems y fue aplicada a 1193 alumnos de segundo de primaria a nivel nacional seleccionados mediante un muestreo poli-etápico por conglomerados y estratificado. En una primera etapa se seleccionaron al azar un conjunto de colegios (conglomerados) dentro de cada estrato. En una segunda etapa, en caso de haber más de 30 alumnos en el grado evaluado, se seleccionaron al azar 30 estudiantes (unidad muestral primaria) para rendir la prueba (ver Anexo). 
En el Cuadro 1 mostramos los análisis de la dificultad y discriminación de los ítems, utilizando la Teoría Clásica de los Tests. Luego presentamos los resultados del análisis factorial a nivel de ítems, utilizando el paquete estadístico SPSS 13. Este programa informático aplica como opción por defecto las correlaciones de Pearson ( $p h i)$ para este tipo de ítems.

\section{Cuadro 1}

Dificultad, discriminación y confiabilidad de los 21 items

\begin{tabular}{ccc}
\hline Item & $p$ & $r_{i t c}$ \\
$\mathrm{~m} 2 \mathrm{p} 021$ & 0,32 & 0,55 \\
$\mathrm{~m} 2 \mathrm{p} 022$ & 0,48 & 0,34 \\
$\mathrm{~m} 2 \mathrm{p} 023$ & 0,64 & 0,52 \\
$\mathrm{~m} 2 \mathrm{p} 024$ & 0,69 & 0,32 \\
$\mathrm{~m} 2 \mathrm{p} 025$ & 0,33 & 0,52 \\
$\mathrm{~m} 2 \mathrm{p} 026$ & 0,15 & 0,37 \\
$\mathrm{~m} 2 \mathrm{p} 027$ & 0,37 & 0,43 \\
$\mathrm{~m} 2 \mathrm{p} 028$ & 0,28 & 0,52 \\
$\mathrm{~m} 2 \mathrm{p} 029$ & 0,22 & 0,39 \\
$\mathrm{~m} 2 \mathrm{p} 030$ & 0,37 & 0,42 \\
$\mathrm{~m} 2 \mathrm{p} 031$ & 0,79 & 0,49 \\
$\mathrm{~m} 2 \mathrm{p} 032$ & 0,76 & 0,56 \\
$\mathrm{~m} 2 \mathrm{p} 033$ & 0,53 & 0,56 \\
$\mathrm{~m} 2 \mathrm{p} 034$ & 0,24 & 0,47 \\
$\mathrm{~m} 2 \mathrm{p} 035$ & 0,20 & 0,42 \\
$\mathrm{~m} 2 \mathrm{p} 036$ & 0,53 & 0,58 \\
$\mathrm{~m} 2 \mathrm{p} 037$ & 0,40 & 0,44 \\
$\mathrm{~m} 2 \mathrm{p} 038$ & 0,41 & 0,53 \\
$\mathrm{~m} 2 \mathrm{p} 039$ & 0,54 & 0,59 \\
$\mathrm{~m} 2 \mathrm{p} 040$ & 0,19 & 0,50 \\
\hline $\mathrm{m} 2 \mathrm{p} 041$ & 0,57 & 0,60 \\
$\dot{a}=0,88$ & & \\
$n=1193$ & &
\end{tabular}

Tal como podemos observar en este cuadro, los índices de dificultad de los ítems fluctúan entre 0,15 y 0,79. Esto nos permite concluir que hay una buena dispersión de los ítems en función 
a su dificultad. Además todas las correlaciones ítem-test corregidas son superiores a 0,30. Esto nos habla de la buena discriminación de los ítems. Finalmente, observamos que el coeficiente alpha de Cronbach tiene un valor de 0,88 que puede ser considerado alto. Es decir, las puntuaciones obtenidas con este instrumento presentan una buena confiabilidad.

\section{Cuadro 2}

Medidas de adecuación de las variables al análisis factorial

Medida de adecuación muestralde

\begin{tabular}{lcc}
\hline \multicolumn{2}{c}{ Kaiser-Meyer-Olkin } & 0,937 \\
& Chi-cuadrado & \\
Prueba de Esfericidad & aproximado & 6345,271 \\
de Bartlett & gl & 210 \\
\hline & Sig. &, 000
\end{tabular}

\section{Cuadro 3}

Resultados de la extracción inicial con el método de ejes principales

Autovalores iniciales

Factor
Suma de las saturaciones al cuadrado de la extracción

\begin{tabular}{|c|c|c|c|c|c|c|}
\hline & Total & $\%$ de la & $\%$ & & $\%$ de la & $\%$ \\
\hline & & varianza & acumulado & Total & varianza & acumulado \\
\hline 1 & 6,383 & 30,4 & 30,4 & 5,749 & 27,4 & 27,4 \\
\hline 2 & 1,445 & 6,9 & 37,3 & 0,864 & 4,1 & 31,5 \\
\hline 3 & 1,009 & 4,8 & 42,1 & 0,376 & 1,8 & 33,3 \\
\hline 4 & 0,988 & 4,7 & 46,8 & & & \\
\hline 5 & 0,920 & 4,4 & 51,2 & & & \\
\hline 6 & 0,885 & 4,2 & 55,4 & & & \\
\hline 7 & 0,833 & 4,0 & 59,3 & & & \\
\hline 8 & 0,813 & 3,9 & 63,2 & & & \\
\hline 9 & 0,767 & 3,7 & 66,9 & & & \\
\hline 10 & 0,707 & 3,4 & 70,2 & & & \\
\hline 11 & 0,692 & 3,3 & 73,5 & & & \\
\hline 12 & 0,674 & 3,2 & 76,7 & & & \\
\hline 13 & 0,661 & 3,1 & 79,9 & & & \\
\hline 14 & 0,632 & 3,0 & 82,9 & & & \\
\hline
\end{tabular}


La unidimensionalidad de un instrumento de medición

Cuadro 3 (Continuación)

\begin{tabular}{cccccc}
\hline \multicolumn{2}{c}{ Autovalores iniciales } & \multicolumn{3}{c}{$\begin{array}{c}\text { Suma de las saturaciones } \\
\text { al cuadrado de la extracción }\end{array}$} \\
Factor & Total & $\begin{array}{c}\text { \% de la } \\
\text { varianza }\end{array}$ & $\begin{array}{c}\% \\
\text { acumulado }\end{array}$ & Total & $\begin{array}{c}\% \text { de la } \\
\text { varianza acumulado }\end{array}$ \\
\hline 15 & 0,604 & 2,9 & 85,8 & & \\
16 & 0,584 & 2,8 & 88,6 & \\
17 & 0,539 & 2,6 & 91,1 & \\
18 & 0,511 & 2,4 & 93,6 & \\
19 & 0,501 & 2,4 & 95,9 & \\
20 & 0,454 & 2,2 & 98,1 & \\
\hline 21 & 0,397 & 1,9 & 100,0 & \\
Total & 21,000 & 100,0 & &
\end{tabular}

El Cuadro 2 nos muestra qué tanto se adecuan nuestras variables al análisis factorial. La medida de adecuación muestral de KMO indica qué proporción de la varianza en las variables es considerada varianza común (causada por factores subyacentes). El valor obtenido $(0,937)$ es bastante alto, por lo cual podemos considerar que sí es posible factorizar esta matriz de correlaciones. La Prueba de Esfericidad de Bartlett contrasta la hipótesis referida a que la matriz de correlaciones con la cual trabajamos es una matriz identidad ${ }^{2}$. El valor chi-cuadrado aproximado es de 6345,271 , que para 210 grados de libertad tiene un nivel de significancia de 0,000. Estos resultados nos permiten rechazar la hipótesis nula al $99 \%$, afirmando que nuestra matriz de correlaciones no corresponde a una matriz de identidad. En conclusión, nuestra matriz de correlaciones puede ser factorizada.

Posteriormente observamos en el Cuadro 3 los autovalores extraídos para cada uno de los factores. Se puede apreciar que tres de ellos son superiores a 1,00 y que en conjunto explican el $42,1 \%$ de la varianza. Luego de la extracción inicial y la aplica-

2 Una matriz identidad implicaría que las variables no se encuentran relacionadas entre sí. 
ción de la extracción de varianza utilizando el método del eje principal, sólo el primer factor posee una saturación cuadrada superior a 1,00 .

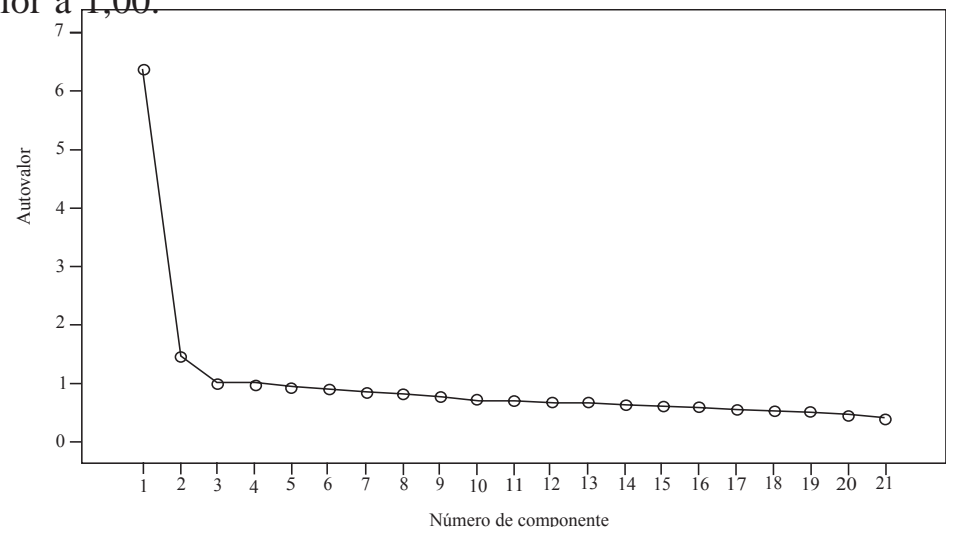

Figura 1. Scree Plot para los 21 ítems, correlaciones phi.

Como se aprecia en la Figura 1, el Scree Plot de Cattell muestra que hay evidencias suficientes para optar por una solución factorial de tipo unidimensional. Sin embargo, la cantidad de varianza explicada por ese factor dominante sólo representa el 27,3\% de la varianza total. Este criterio resulta insuficiente para sustentar la unidimensionalidad de la escala según lo propuesto por Carmines y Zeller (1979).

¿Qué ocurre cuando factorizamos los mismos ítems, pero utilizando la matriz de correlaciones tetracóricas en lugar de las correlaciones phi? Para responder a esta pregunta, mostramos los resultados en el Cuadro 4. 
La unidimensionalidad de un instrumento de medición

\section{Cuadro 4}

Medidas de adecuación de las variables al análisis factorial

\begin{tabular}{ccc}
\hline \multicolumn{3}{c}{ Medida de adecuación muestralde } \\
Kaiser-Meyer-Olkin & 0,925 \\
\hline & Chi-cuadrado & \\
Prueba de Esfericidad & aproximado & 15715,686 \\
de Bartlett & gl & 210 \\
& Sig. &, 000 \\
\hline
\end{tabular}

\section{Cuadro 5}

Resultados de la extracción inicial con el método de ejes principales

\begin{tabular}{|c|c|c|c|c|c|c|}
\hline \multirow[b]{2}{*}{ Factor } & \multicolumn{3}{|c|}{ Autovalores iniciales } & \multicolumn{3}{|c|}{$\begin{array}{l}\text { Sumas de las saturaciones } \\
\text { al cuadrado de la extracción }\end{array}$} \\
\hline & Total & $\begin{array}{l}\% \text { de la } \\
\text { varianza }\end{array}$ & $\begin{array}{c}\% \\
\text { acumulado }\end{array}$ & Total & $\begin{array}{l}\% \text { de la } \\
\text { varianza }\end{array}$ & $\begin{array}{c}\% \\
\text { acumulado }\end{array}$ \\
\hline 1 & 10,088 & 48,036 & 48,036 & 9,659 & 45,994 & 45,994 \\
\hline 2 & 1,246 & 5,932 & 53,969 &, 873 & 4,157 & 50,151 \\
\hline 3 & 1,008 & 4,799 & 58,768 & ,542 & 2,583 & 52,734 \\
\hline 4 & ,921 & 4,384 & 63,152 & & & \\
\hline 5 & ,866 & 4,125 & 67,277 & & & \\
\hline 6 & 809 & 3,854 & 71,130 & & & \\
\hline 7 & ,712 & 3,390 & 74,520 & & & \\
\hline 8 & ,666 & 3,172 & 77,692 & & & \\
\hline 9 & ,608 & 2,896 & 80,589 & & & \\
\hline 10 &, 530 & 2,522 & 83,111 & & & \\
\hline 11 & ,500 & 2,381 & 85,492 & & & \\
\hline 12 & ,486 & 2,313 & 87,805 & & & \\
\hline 13 & ,429 & 2,043 & 89,848 & & & \\
\hline 14 & ,407 & 1,938 & 91,786 & & & \\
\hline 15 & 357 & 1,702 & 93,488 & & & \\
\hline 16 & ,340 & 1,621 & 95,110 & & & \\
\hline 17 & ,288 & 1,370 & 96,480 & & & \\
\hline 18 & 259 & 1,233 & 97,713 & & & \\
\hline 19 & ,227 & 1,082 & 98,795 & & & \\
\hline 20 & , 141 & ,673 & 99,468 & & & \\
\hline 21 &, 112 &, 532 & 100,000 & & & \\
\hline Total & 21,00 & 100,00 & & & & \\
\hline
\end{tabular}


Al examinar si la matriz de correlaciones tetracóricas es adecuada para realizar un análisis factorial, vemos en el Cuadro 4 que el valor del índice KMO $(0,925)$ es bastante alto. La Prueba de Esfericidad de Bartlett posee un valor chi-cuadrado aproximado de 15715,686 que para 210 grados de libertad tiene un nivel de significancia de 0,000. En conclusión, nuestra matriz de correlaciones puede ser factorizada.

Posteriormente observamos en el Cuadro 5 los autovalores extraídos para cada uno de los factores. Se puede apreciar que tres de ellos son superiores a 1,00 y que en conjunto explican el $58,8 \%$ de la varianza. Luego de la extracción inicial y la aplicación de la extracción de varianza utilizando el método del eje principal, sólo el primer factor posee una saturación cuadrada superior a 1,00 .

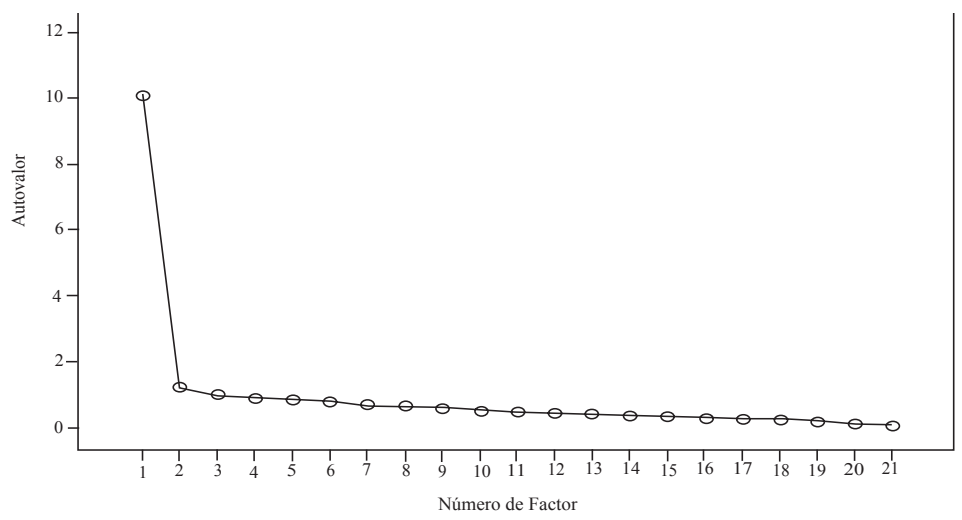

Figura 2. Scree Plot para los 21 ítems, correlaciones tetracóricas.

Como se aprecia en la Figura 2, hay evidencias suficientes para suponer que se puede optar por una solución factorial de tipo unidimensional. Este único factor dominante explica casi el $46,0 \%$ de la varianza. Este criterio resulta suficiente para sustentar la unidimensionalidad de la escala según lo propuesto por Carmines y Zeller (1979). 


\section{Ejemplo para items politómicos}

Presentaremos los análisis factoriales realizados a la subescala Respuesta Cognitiva de Estrés construida por Burga (1999). Dicha subescala está conformada por 21 ítems y fue aplicada a 480 alumnos de una universidad privada de Lima Metropolitana. Al realizar algunos análisis posteriores, se decidió eliminar 3 ítems, lo cual mejoró su consistencia interna.

En el Cuadro 6 mostramos los análisis de la discriminación de los ítems utilizando la Teoría Clásica de los Tests. Luego presentamos los resultados del análisis factorial al nivel de ítems, utilizando el paquete estadístico SPSS 13 (Ver Anexo).

\section{Cuadro 6}

Dificultad, discriminación y confiabilidad de los 19 items

\begin{tabular}{|c|c|}
\hline Item & $r_{i t c}$ \\
\hline $\mathrm{c} 001$ & 0,39 \\
\hline $\mathrm{c} 005$ & 0,64 \\
\hline $\mathrm{c} 008$ & 0,59 \\
\hline $\mathrm{c} 009$ & 0,51 \\
\hline $\mathrm{c} 012$ & 0,41 \\
\hline $\mathrm{c} 018$ & 0,61 \\
\hline c019 & 0,41 \\
\hline $\mathrm{c} 022$ & 0,62 \\
\hline $\mathrm{c} 023$ & 0,52 \\
\hline $\mathrm{c} 030$ & 0,38 \\
\hline $\mathrm{c} 040$ & 0,47 \\
\hline $\mathrm{c} 044$ & 0,67 \\
\hline $\mathrm{c} 045$ & 0,71 \\
\hline c049 & 0,69 \\
\hline $\mathrm{c} 057$ & 0,70 \\
\hline c059 & 0,71 \\
\hline $\mathrm{c} 060$ & 0,64 \\
\hline c061 & 0,62 \\
\hline $\mathrm{c} 062$ & 0,60 \\
\hline
\end{tabular}


Como podemos observar en el Cuadro 6, todas las correlaciones ítem-test corregidas son superiores a 0,30. Esto nos habla de la buena discriminación de los ítems. El coeficiente alpha de Cronbach tiene un valor de 0,92 que puede ser considerado alto. Es decir, las puntuaciones obtenidas con este instrumento presentan una buena confiabilidad.

\section{Cuadro 7}

Medidas de adecuación de las variables al análisis factorial

\begin{tabular}{|c|c|c|}
\hline \multicolumn{2}{|c|}{$\begin{array}{c}\text { Medida de adecuación muestral de } \\
\text { Kaiser-Meyer-Olkin }\end{array}$} & 0,929 \\
\hline $\begin{array}{c}\text { Prueba de Esfericidad } \\
\text { de Bartlett }\end{array}$ & $\begin{array}{c}\text { Chi-cuadrado } \\
\text { aproximado } \\
\text { gl } \\
\text { Sig. }\end{array}$ & $\begin{array}{c}4254,712 \\
171 \\
0,000\end{array}$ \\
\hline
\end{tabular}

Vemos en el Cuadro 7 que la medida de adecuación muestral de KMO posee un valor bastante alto $(0,929)$, por lo cual podemos considerar que sí es posible factorizar esta matriz de correlaciones. La Prueba de Esfericidad de Bartlett señala que el valor chi-cuadrado aproximado es de 4254,712, que para 171 grados de libertad tiene un nivel de significancia de 0,000. Estos resultados nos permiten rechazar la hipótesis nula al 99\%, afirmando que nuestra matriz de correlaciones no corresponde a una matriz de identidad. En conclusión, nuestra matriz de correlaciones puede ser factorizada. 
La unidimensionalidad de un instrumento de medición

\section{Cuadro 8}

Resultados de la extracción inicial con el método de ejes principales

\begin{tabular}{ccccccc}
\hline \multicolumn{3}{c}{ Autovalores iniciales } & \multicolumn{3}{c}{$\begin{array}{c}\text { Sumas de las saturaciones } \\
\text { al cuadrado de la extracción }\end{array}$} \\
Factor & Total & $\begin{array}{c}\text { \% de la } \\
\text { varianza }\end{array}$ & $\begin{array}{c}\% \\
\text { acumulado }\end{array}$ & Total & $\begin{array}{c}\text { varianza } \\
\text { vacumulado }\end{array}$ & acta \\
\hline 1 & 7,710 & 40,580 & 40,580 & 7,228 & 38,040 & 38,040 \\
2 & 1,884 & 9,917 & 50,497 & 1,433 & 7,542 & 45,582 \\
3 & 1,312 & 6,906 & 57,403 &, 771 & 4,057 & 49,639 \\
4 &, 955 & 5,028 & 62,431 & & & \\
5 &, 770 & 4,055 & 66,486 & & & \\
6 &, 682 & 3,592 & 70,077 & & & \\
7 &, 629 & 3,312 & 73,389 & & & \\
8 &, 602 & 3,167 & 76,556 & & & \\
9 &, 565 & 2,972 & 79,528 & & & \\
10 &, 518 & 2,728 & 82,256 & & & \\
11 &, 492 & 2,589 & 84,845 & & & \\
12 &, 477 & 2,511 & 87,356 & & & \\
13 &, 424 & 2,229 & 89,585 & & & \\
14 &, 402 & 2,114 & 91,699 & & & \\
15 &, 381 & 2,003 & 93,702 & & & \\
16 &, 368 & 1,939 & 95,641 & & & \\
17 &, 323 & 1,702 & 97,344 & & & \\
18 &, 276 & 1,452 & 98,795 & & & \\
19 &, 229 & 1,205 & 100,000 & & & \\
\hline Total & 19,0 & 100,0 & & & & \\
\hline
\end{tabular}

Posteriormente observamos en el Cuadro 8 los autovalores extraídos para cada uno de los factores. Se puede apreciar que tres de ellos son superiores a 1,00 y que en conjunto explican el 49,6\% de la varianza. Luego de la extracción inicial y la aplicación de la extracción de varianza utilizando el método del eje principal, sólo el primer y segundo factor poseen una saturación cuadrada superior a 1,00 . 


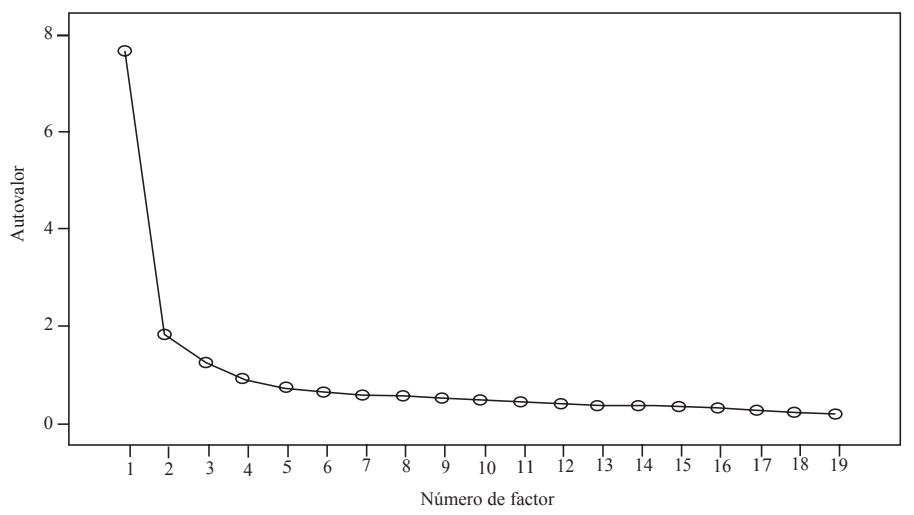

Figura 3. Scree Plot para los 19 ítems.

Como se aprecia en la Figura 3, el Scree Plot de Cattell muestra que hay evidencias suficientes para optar por una solución factorial de tipo unidimensional. Sin embargo, la cantidad de varianza explicada por ese factor dominante sólo representa el 38,0\% de la varianza total. Este criterio resulta insuficiente para sustentar la unidimensionalidad de la escala según lo propuesto por Carmines y Zeller (1979).

Tal y como hicimos con los ítems dicotómicos de la prueba de matemáticas, utilizaremos otra matriz de correlaciones para volver a factorizar los ítems de esta subescala de Respuesta Cognitiva al Estrés. 
La unidimensionalidad de un instrumento de medición

\section{Cuadro 9}

Medidas de adecuación de las variables al análisis factorial

\begin{tabular}{|c|c|c|}
\hline \multicolumn{2}{|c|}{$\begin{array}{l}\text { Medida de adecuación muestral de } \\
\text { Kaiser-Meyer-Olkin }\end{array}$} & 0,923 \\
\hline $\begin{array}{l}\text { Prueba de Esfericidad } \\
\text { de Bartlett }\end{array}$ & $\begin{array}{c}\text { Chi-cuadrado } \\
\text { aproximado } \\
\text { gl } \\
\text { Sig. }\end{array}$ & $\begin{array}{c}5401,528 \\
171 \\
0,000\end{array}$ \\
\hline
\end{tabular}

\section{Cuadro 10}

Resultados de la extracción inicial con el método de ejes principales

\begin{tabular}{ccccccc}
\hline \multicolumn{3}{c}{ Autovalores iniciales } & \multicolumn{3}{c}{$\begin{array}{c}\text { Sumas de las saturaciones } \\
\text { al cuadrado de la extracción }\end{array}$} \\
Factor & Total & $\begin{array}{c}\text { \% de la } \\
\text { varianza }\end{array}$ & $\begin{array}{c}\text { acumulado } \\
\text { acta }\end{array}$ & $\begin{array}{c}\text { Total } \\
\text { varianza }\end{array}$ & acumulado \\
\hline 1 & 8,546 & 44,981 & 44,981 & 8,126 & 42,767 & 42,767 \\
2 & 1,963 & 10,333 & 55,314 & 1,576 & 8,296 & 51,063 \\
3 & 1,330 & 7,001 & 62,316 &, 838 & 4,408 & 55,471 \\
4 &, 943 & 4,961 & 67,277 & & & \\
5 &, 737 & 3,880 & 71,158 & & & \\
6 &, 648 & 3,411 & 74,569 & & & \\
7 &, 578 & 3,040 & 77,609 & & & \\
8 &, 556 & 2,927 & 80,536 & & & \\
9 &, 494 & 2,600 & 83,136 & & & \\
10 &, 457 & 2,407 & 85,543 & & & \\
11 &, 434 & 2,282 & 87,825 & & & \\
12 &, 420 & 2,209 & 90,034 & & & \\
13 &, 362 & 1,907 & 91,941 & & & \\
14 &, 328 & 1,728 & 93,669 & & & \\
15 &, 311 & 1,637 & 95,306 & & & \\
16 &, 298 & 1,568 & 96,874 & & & \\
17 &, 244 & 1,282 & 98,156 & & & \\
18 &, 203 & 1,066 & 99,223 & & & \\
19 &, 148 &, 777 & 100,000 & & & \\
\hline Total & 19,0 & 100,0 & & & & \\
\hline
\end{tabular}


Vemos en el Cuadro 9 que la medida de adecuación muestral de KMO posee un valor bastante alto $(0,923)$, por lo cual podemos considerar que sí es posible factorizar esta matriz de correlaciones. La Prueba de Esfericidad de Bartlett señala que el valor chi-cuadrado aproximado es de 5401,528, que para 171 grados de libertad tiene un nivel de significancia de 0,000. Estos resultados nos permiten rechazar la hipótesis nula al 99\%, afirmando que nuestra matriz de correlaciones no corresponde a una matriz de identidad. En conclusión, nuestra matriz de correlaciones puede ser factorizada.

Luego observamos en el Cuadro 10 los autovalores iniciales extraídos para cada uno de los factores. Se puede apreciar que tres de ellos son superiores a 1,00 y que en conjunto explican el $62,3 \%$ de la varianza. Luego de la aplicación de la extracción de varianza utilizando el método del eje principal, sólo el primer y segundo factor poseen una saturación cuadrada superior a 1,00. Ambos explican en conjunto el 51,1\% de la varianza.

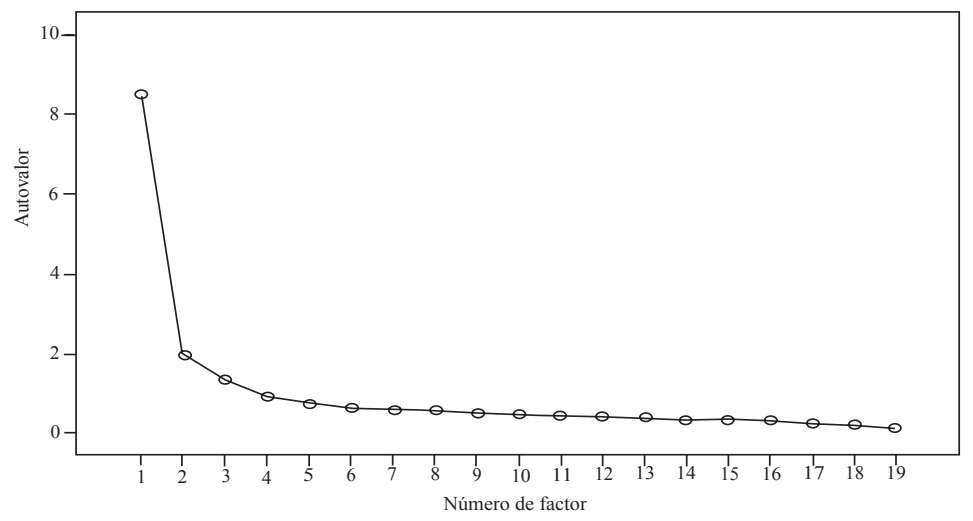

Figura 4. Scree Plot para los 19 ítems, correlaciones policóricas. 
La Figura 4 muestra que hay evidencias suficientes para suponer que se puede optar por una solución factorial de tipo unidimensional. Este factor dominante explica el 42,3\% de la varianza, criterio que resulta suficiente según lo propuesto por Carmines y Zeller (1979).

\section{Conclusiones}

Hemos visto que, para el mismo conjunto de datos, en el caso de utilizar correlaciones $p h i$, subestimamos la cantidad de varianza común compartida. Esto nos llevaría a rechazar el supuesto de unidimensionalidad de esta escala de medición. Cuando utilizamos las matrices de correlaciones tetracóricas para realizar el análisis factorial, pudimos aceptar el supuesto de unidimensionalidad de la escala. Estos mismos datos han mostrado un buen ajuste a un modelo de análisis Rasch para Ítems Dicotómicos, que nos provee de otros índices (el outfit y el análisis de componentes principales basado en los residuos) a fin de analizar la unidimensionalidad de los ítems. Estos índices también dan evidencias a favor de la unidimensionalidad de estos ítems.

Por otra parte, cuando factorizamos los datos provenientes de ítems politómicos utilizando las matrices de correlaciones de Pearson, observamos que el primer factor extraído no cumple con el criterio propuesto por Carmines y Zeller (1979) para considerar una escala como unidimensional. Igual que en el caso de los ítems dicotómicos, utilizamos como matriz a factorizar aquella basada en las correlaciones policóricas. En este caso el primer factor extraído sí es suficiente para sustentar que se trata de una escala fundamentalmente unidimensional. Asimismo, los resultados del Análisis Rasch con el modelo de Andrich apoyan la unidimensionalidad de nuestros ítems politómicos. 


\section{Referencias}

Anderson, J., Gerbin, D. \& Hunter, J. (1987). On the assessment of unidimensional measurement: Internal and external consistency, and overall consistency criteria. Journal of Marketing Research, 24(4), 432-437.

Bejar, I. (1983). Achievement testing: Recent advances. California: Sage.

Burga, A. (1999). Construcción, confiabilidad y validez de la Escala de Respuesta al Estrés MNC. Tesis de licenciatura, Universidad de Lima.

Carmines, E. \& Zeller, R. (1979). Reliability and validity assessment. California: Sage.

Cuesta, M. (1996). Unidimensionalidad. En J. Muñiz (Ed.), Psicometría. Madrid: Pirámide.

Embretson, S. \& Reise, S. (2000). Item Response Theory for psychologists. Nueva Jersey: Lawrence Erlbaum.

Guilford, P. \& Fruchter, B. (1984). Estadística aplicada a la psicología y la educación. México: McGraw-Hill.

Hambleton, R., Swaminathan, W. \& Rogers, J. (1991). Fundamentals of Item Response Theory. California: Sage.

Hattie, J. (1985). Methodology review: Assessing unidimensionality of tests and items. Applied Psychological Measurement, 9(2), 139-164.

Henry, D. \& Alred, K. (1997). Diagnosing measure covariance. Rasch Measurement Transactions, 11(1), 556.

Jiménez, J., Artiles, C. \& Yáñez, G. (1997). Creencias de los profesores sobre la enseñanza de la lectura. IberPsicología, 2(2). Recuperado el 18 de agosto de 2004, de http://fsmorente.filos.ucm.es/publicaciones/iberpsicologia/IberPsi3/ Jimenez/Jimenez.htm

Jöreskog, K. (2002). Structural Equation Modeling with ordinal variables using LISREL. Scientific Software International. 
Recuperado el 1 de octubre de 2004, de http://www.ssicentral. com/lisrel/ordinal.pdf

Kubinger, K. (2003). On artificial results due to using factor analysis for dichotomous variables. Psychology Science, 45(1), 106-110.

Linacre, J. (1994). DIMTEST diminuendo. Rasch Measurement Transactions, 8(3), 384.

Merende, P. (1997). A guide to the proper use of factor analysis in the conduct and reporting of research: Pitfalls to avoid. Measurement and Evaluation in Counseling and Development, 30(3), 156-164.

Moosbrugger, H. \& Hartig, J. (2002). Factor analysis in personality research: Some artifacts and their consequences for psychological assessment. Psychologische Beiträge, 44(1), 136-158.

Muñiz, J. (1997). Introducción a la Teoría de Respuesta a los Ítems. Madrid: Pirámide.

Nunnally, J. \& Bernstein, I. (1995). Teoría psicométrica (2a . ed.). México: McGraw-Hill.

O'Connor, B. (s. f.) Cautions regarding item-level factor analyses. Recuperado el 29 de septiembre de 2004, de http:// flash.lakeheadu.ca/ boconno2/itemanalysis.html

Reckase, M. (1979). Unifactor latent trait models applied to multifactor tests: Results and implications. Journal of Educational Statistics, 4(3), 207-230.

Sun, P., Dailey, R. \& Lemus, D. (2002). The use of exploratory factor analysis and principal components analysis in communications research. Human Communication Research, 28(4), 562-577.

Waller, N. (2001). MicroFACT 2.0. A Microcomputer Factor Analysis Program for ordered polytomous data and mainframe size problems. St. Paul, MN: Assessment Systems Corporation.

Wright, B. (1994). Unidimensionality coefficient. Rasch Measurement Transactions, 8(3), 385.

Wright, B. \& Linacre, J. (1989). MESA research memorandum 44. Archives of Physical Medicine and Rehabilitation, 70(12), 857860. 
Wright, B. \& Masters, G. (1982). Rating scale analysis. Chicago: Mesa Press.

Wright, B. \& Stone, M. (1998). Diseño de mejores pruebas. México: CENEVAL.

Zwick, R. (1985). Assessment of the dimensionality of NAEP year 15 reading data (ETS Res. Rep. No. 86-4). Princeton: Educational Testing Service. 
La unidimensionalidad de un instrumento de medición

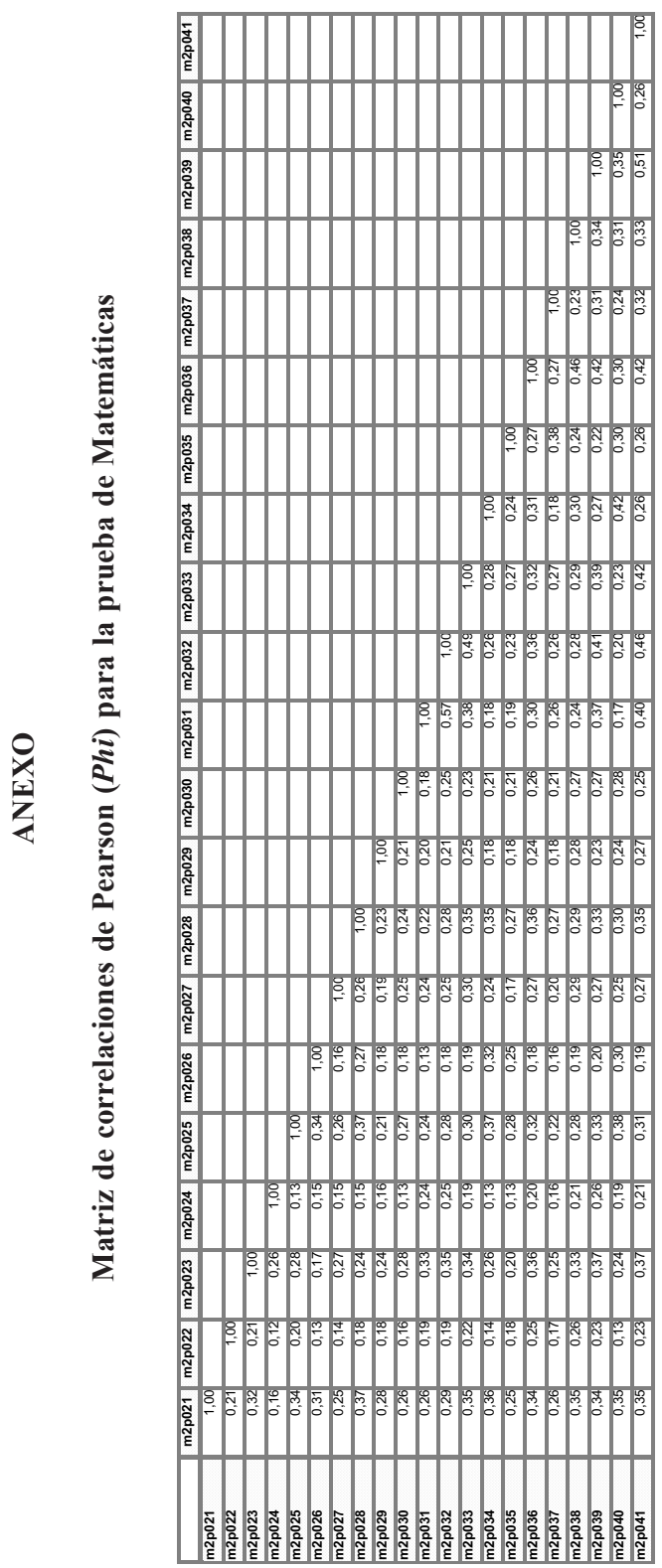


Andrés Burga León

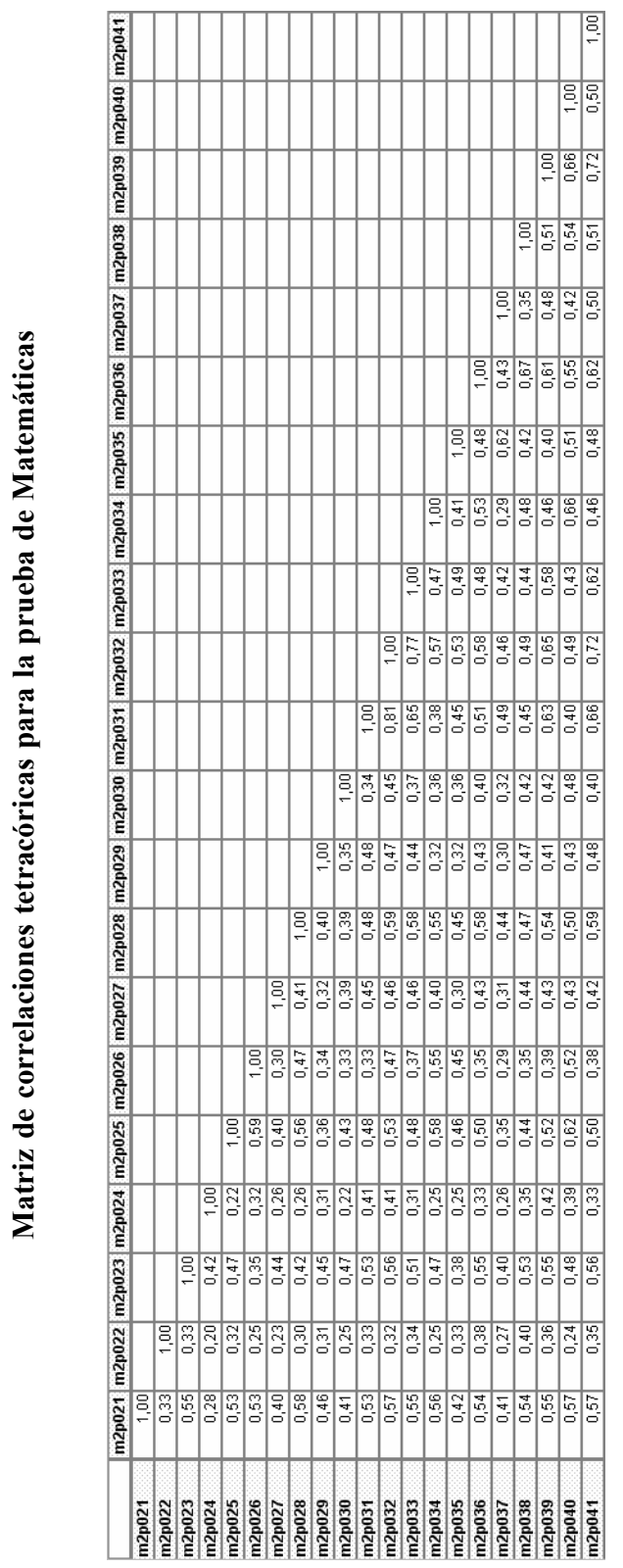


La unidimensionalidad de un instrumento de medición

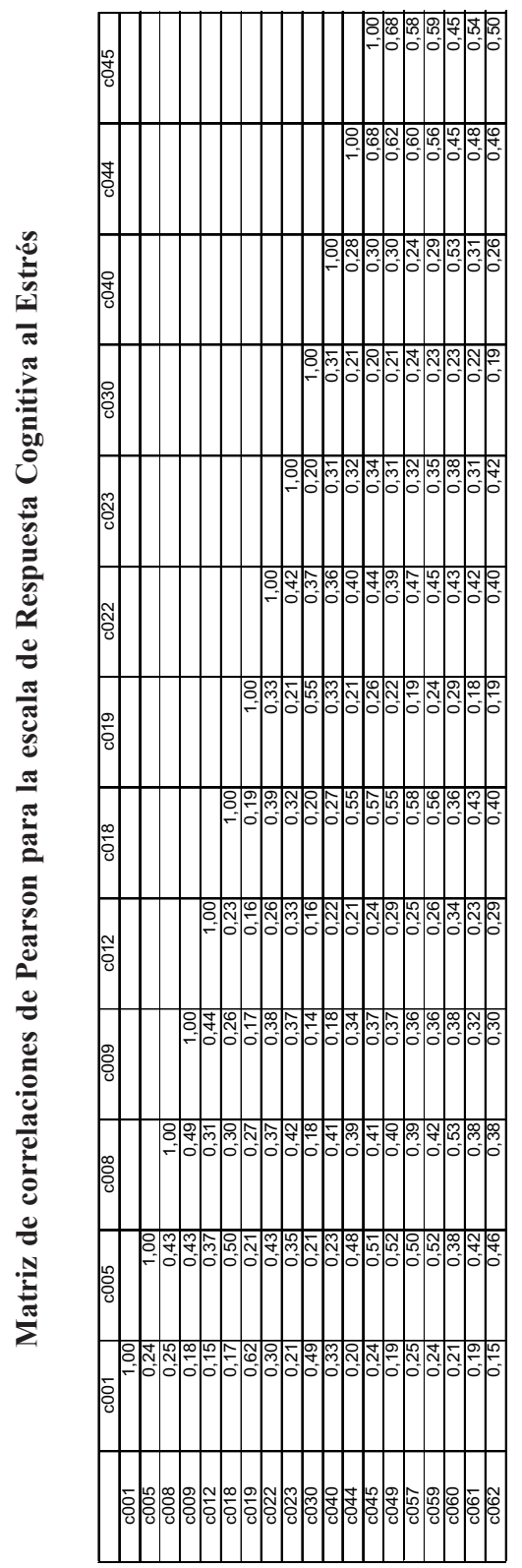


Andrés Burga León

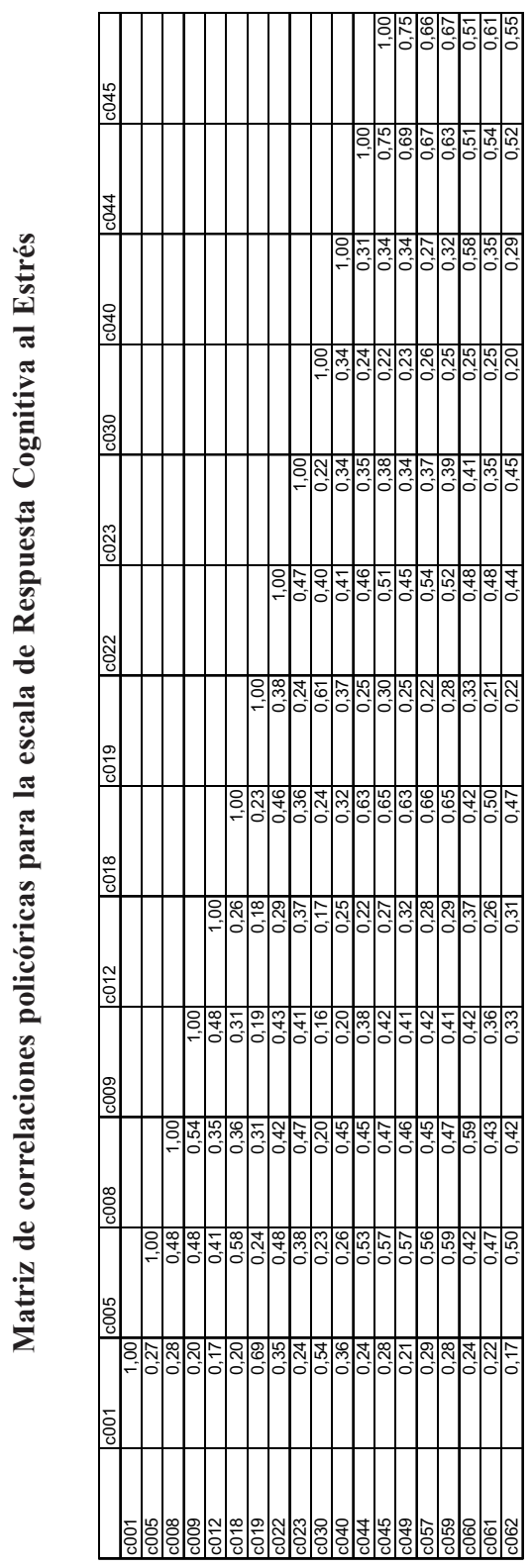

